\title{
Supporting Information for Far-Red Emission of mPlum Fluorescent Protein Results from Excited-State Interconversion Between Chromophore Hydrogen-Bonding States
}

\author{
Eunjin Yoon ${ }^{1}$, Patrick E. Konold ${ }^{2}$, Junghwa Lee ${ }^{1}$, Taiha Joo $^{1 *}$ and Ralph Jimenez ${ }^{2,3^{*}}$ \\ ${ }^{1}$ Department of Chemistry, Pohang University of Science and Technology, \\ Pohang, South Korea 790-784 \\ 2 JILA, 440 UCB University of Colorado and NIST, Boulder, CO 80309-0440 \\ ${ }^{3}$ Department of Chemistry \& Biochemistry, 215 UCB, University of Colorado, Boulder, CO \\ 80309-0215
}

Contents

1. Description of the global fitting method

2. Species-associated spectra from decay-associated spectra

3. Selected single-wavelength TRF transients and fits for mPlum

4. Fluorescence lifetime of mPlum measured by TCSPC

5. TRANES of E16H

6. Global fit of E16H TRFS

7. Time-resolved fluorescence anisotropy of mPlum

8. Complete author name list for references $6 \& 7$. 


\section{Description of the global fitting method:}

Transients at each wavelength were nonlinear least-squares fitted to a sum of exponential functions convoluted with an instrument response function, which was assumed to be a Gaussian with a standard deviation of $70 \mathrm{fs}$ determined by the cross correlation between the gate and scattered pump pulse. Amplitudes $A(\lambda)$ are assumed to be wavelength-dependent with a common set of time constants that applies across the spectra (Equation S1):

$$
\mathrm{I}(\lambda, \mathrm{t})=\sum_{\mathrm{j}} \mathrm{A}(\lambda) \mathrm{e}^{-\mathrm{t} / \tau_{\mathrm{j}}}
$$

Initial values for the amplitudes were all set to the amplitude at each wavelength at time zero.
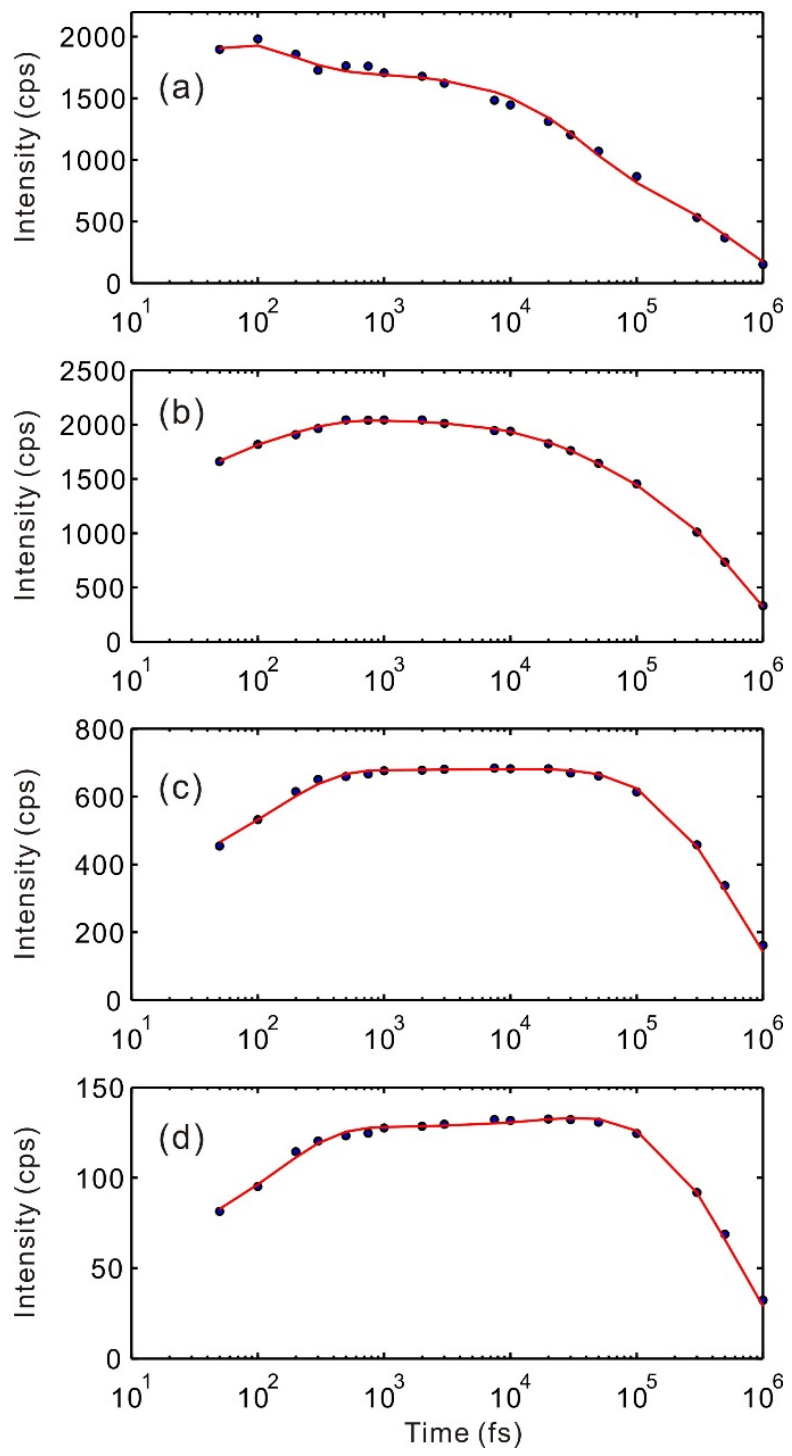

Figure S1. Selected single-wavelength transients and fits of mPlum at the frequencies of (a) 15870, (b) 15270, (c) 14490, and (d) $13510 \mathrm{~cm}^{-1}$. 


\section{Species associated spectra (SAS)}

The simple kinetic model excluding the initial ultrafast $160 \mathrm{fs}$ component, can be expressed by a two state interconversion process shown below with both $A$ and $B$ populated at time zero, where $A$ and $B$ are the direct hydrogen bonded and $\mathrm{H}_{2} \mathrm{O}$-mediated hydrogen bonded species in the excited electronic states, respectively, and $A_{G}$ and $B_{G}$ represent the corresponding ground states.

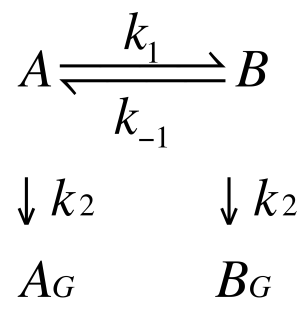

The rate equations are

$$
\begin{aligned}
& \frac{d[\mathrm{~A}]}{d t}=-\left(k_{1}+k_{2}\right)[\mathrm{A}]+k_{-1}[\mathrm{~B}] \\
& \frac{d[\mathrm{~B}]}{d t}=k_{1}[\mathrm{~A}]-\left(k_{-1}+k_{3}\right)[\mathrm{B}]
\end{aligned}
$$

Because only a single 610 ps component was observed for all wavelengths at long times, we can assume that $k_{1} \gg k_{2}, k_{3}$. We also neglect $k_{-1}$. Then the kinetic scheme above becomes a simple two-step process with both $A$ and $B$ populated initially, and one obtains time-dependent populations:

$$
\begin{aligned}
A(t) & =A_{0} e^{-k_{1} t} \\
B(t) & =B_{0} e^{-k_{3} t}-A_{0}\left(\frac{k_{1} e^{-k_{1} t}}{k_{1}-k_{3}}-\frac{k_{1} e^{-k_{3} t}}{k_{1}-k_{3}}\right) \\
& \cong\left(1-A_{0}\right) e^{-k_{3} t}-A_{0}\left(e^{-k_{1} t}-e^{-k_{3} t}\right), \quad\left(A_{0}+B_{0}=1\right) \\
& =e^{-k_{3} t}-A_{0} e^{-k_{1} t}
\end{aligned}
$$

The TRF spectra are

$$
\begin{aligned}
I(\omega, t) & =I_{A}(\omega) A_{0} e^{-k_{1} t}+I_{B}(\omega)\left(e^{-k_{3} t}-A_{0} e^{-k_{1} t}\right) \\
& =A_{0}\left(I_{A}(\omega)-I_{B}(\omega)\right) e^{-k_{1} t}+I_{B}(\omega) e^{-k_{3} t}
\end{aligned}
$$

One obtains decay associated spectra (DAS)

$$
\begin{aligned}
& I_{k_{1}}(\omega)=A_{0}\left(I_{A}(\omega)-I_{B}(\omega)\right) \\
& I_{k_{3}}(\omega)=I_{B}(\omega)
\end{aligned}
$$

where $I_{k_{1}}(\omega)$ and $I_{k_{3}}(\omega)$ are the DAS for $k_{1}$ and $k_{3}$ from the global fits. $I_{A}(\omega)$ and $I_{B}(\omega)$ are the SAS, given by

$$
\begin{aligned}
& I_{A}(\omega)=I_{k_{3}}(\omega)+\frac{I_{k_{1}}(\omega)}{A_{0}} . \\
& I_{B}(\omega)=I_{k_{3}}(\omega)
\end{aligned}
$$


Therefore $I_{k_{3}}(\omega)$ ( $\tau_{2}$ component) directly represents $I_{A}(\omega)$ and $I_{B}(\omega)$ can be calculated from the initial populations $\left(A_{0}, B_{0}\right)$. The SAS of $A$ and $B$, assuming $A_{0}=0.72$ are calculated and given in Figure 3.

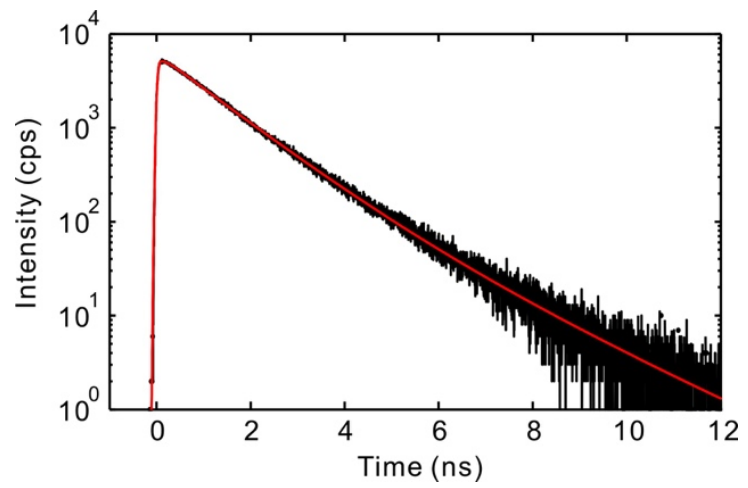

Figure S2. (black) Fluorescence lifetime of mPlum measured by TCSPC, excited at $570 \mathrm{~nm}$, detected at $650 \mathrm{~nm}$. (red) Multiexponential fit to $130 \mathrm{ps}(-17 \%)$ and $1.09 \mathrm{~ns}(75 \%), 1.92 \mathrm{~ns}(8 \%)$.

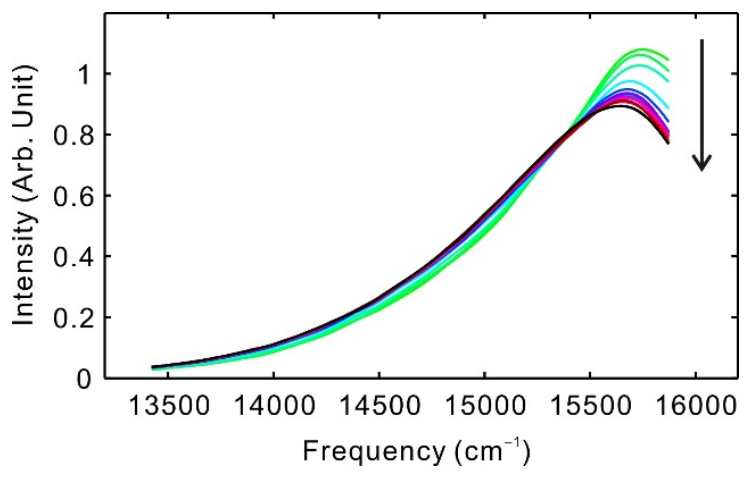

Figure S3. Time-resolved area-normalized emission spectra (TRANES) of mPlum/E16H mutant. Times are 0, 50, 100, 200, 300, 500, 750, $1000 \mathrm{fs}, 2,3,7.5,10,20,30,50,100,300,500$, and $1000 \mathrm{ps}$. Time increases in the direction of the arrow. 


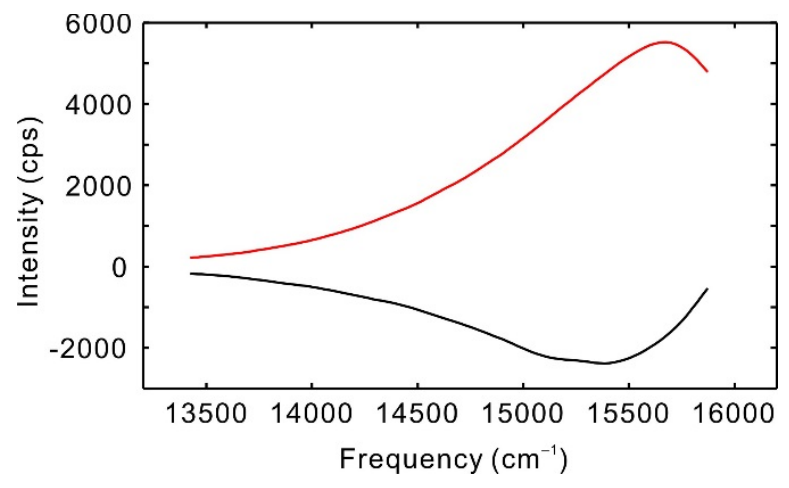

Figure S4. Amplitudes of each component of time-evolution from global analysis of TRFS of mPlum E16H mutant. Black and red lines correspond to $110 \mathrm{fs}$ and 770 ps components, respectively.

\section{Time-Resolved Fluorescence Anisotropy}

Excited state reactive motions such as chromophore cis-trans isomerization or other conformational changes may result in a substantial rotation of the emission dipole moment relative to the absorption dipole. To uncover these changes, we therefore performed timeresolved fluorescence depolarization measurements on mPlum at the peak frequency of the fluorescence spectra to yield the time dependent anisotropy, $\gamma(\mathrm{t})$ according to Equation S2:

$$
\gamma(t)=\frac{I_{\|}(t)-I_{\perp}(t)}{I_{\|}(t)+2 I_{\perp}(t)}
$$

where $I_{\|}$and $I_{\perp}$ are fluorescence intensity with the relative polarizations of the pump and fluorescence parallel and perpendicular, respectively. The calculated $\gamma(t)$ is plotted in Figure S5 and the exponential fitting parameters are given in Table S1.

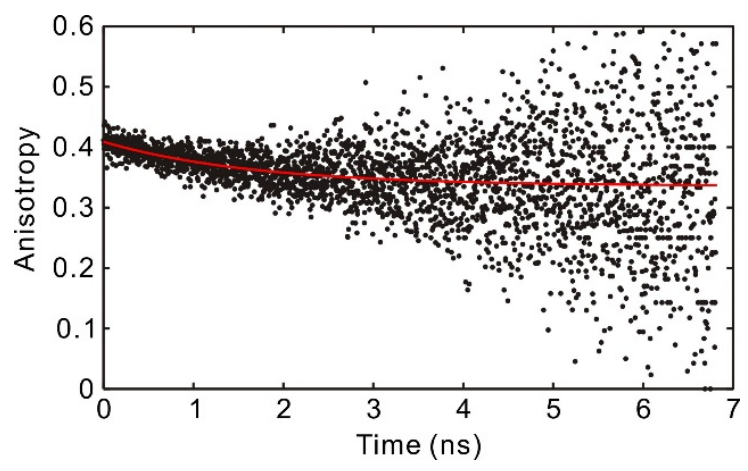

Figure S5. TRF anisotropy transient for mPlum, measured at emission wavelength of $650 \mathrm{~nm}$. The red line represents a fit to exponential functions. Fit parameters are collected in Table 1.

Table S1. Fit parameters describing the TRF anisotropy transient.

\begin{tabular}{cccccc}
\hline & $\gamma_{0}$ & $a$ & $\tau_{\mathbf{r}}(\mathbf{n s})$ & $\gamma_{\infty}$ & $\theta(\mathbf{d e g} .)^{\text {a) }}$ \\
\hline mPlum & 0.409 & 0.07 & 1.7 & 0.336 & 20 \\
\hline
\end{tabular}

a) Angle between the transition dipoles at time zero and long time. 
An intial anisotropy value $\left(\gamma_{0}\right)$ of 0.409 was followed by a decay which fit to a $\tau=1.7$ ns time constant with a terminal value of the anistropy of 0.336 . It seems reasonable that the residual anisotropy $\left(\gamma_{\infty}\right)$ does not decay to zero since mPlum is too large to rotate entirely within the experimental time window. The anisotropy decay time constants associated with rotational reorientation was previously reported as $>10$ ns for monomeric FPs. Rotation angles were calculated from the initial and terminal anisotropies, with the time dependent anisotropy given by

$$
\gamma(t)=\gamma_{0} \frac{3\left\langle\cos ^{2} \theta\right\rangle-1}{2}
$$

We conclude by noting that other processes besides rotation can lead to anisotropy decay. For example, it was reported that dimeric enhanced GFP shows a 1.4 ns anisotropy decay time resulting from excitation transfer between chromophores within the dimer, whereas DsRed shows a 211 ps anisotropy decay time corresponding to excitation transfer within the tetramer. It seems unlikely that excitation transfer is responsible for the depolarization timescales observed for mPlum. We therefore suggest that polarization rotation in mPlum is caused by reorientation of the emission dipole as a result of structural changes within the chromophore binding site.

For References $6 \& 7$, the complete citations, including all authors are:

6. Lin, M. Z.; McKeown, M. R.; Ng, H.-L.; Aguilera, T. A.; Shaner, N. C.; Campbell, R. E.; Adams, S. R.; Gross, L. A.; Ma, W.; Alber, T.; Tsien, R. Y. Autofluorescent Proteins with Excitation in the Optical Window for Intravital Imaging in Mammals. Chemistry \& Biology 2009, 16, 11691179.

7. Chu, J.; Haynes, R. D.; Corbel, S. Y.; Li, P.; Gonzalez-Gonzalez, E.; Burg, J. S.; Ataie, N. J.; Lam, A. J.; Cranfill, P. J.; Baird, M. A.; Davidson, M. W.; Ng, H.-L.; Garcia, K. C.; Contag, C. H.; Shen, K.; Blau, H. M.; Lin, M. Z. Non-Invasive Intravital Imaging of Cellular Differentiation with a Bright Red-Excitable Fluorescent Protein. Nature Methods 2014, 11, 572-578. 